\title{
Development of an Efficient Information Model to Prevent Depression among Hysterectomy Patients
}

\author{
Seong-Ran Lee \\ Department of Medical Information, Kongju National University \\ lsr2626@naver.com
}

\begin{abstract}
The purpose of this study is to analyze the development of an efficient information model to prevent depression among hysterectomy patients. The pairwise t-test was done to the before and after the application for empirical performance rate of a depression prevention program. The results of this paper are follows. First, the mean score of depression status after application significantly decreased than subjects before the application( $t=2.08, p=.015)$. Second, the present research showed that the empirical performance rate of patients with hysterectomy can be increased to 68.5-74.2\% by the model application. Therefore, a new information model can be utilized as an effective intervention for self-management of a depression prevention in hysterectomy patients. In addition, this study will contribute to develop the role of empirical model in comprehensive and systematical prevention and treatment of depression among hysterectomy patients.
\end{abstract}

Keywords: Development, Information, Model, Depression, Prevention, Hysterectomy patients

\section{Introduction}

Cervical cancer is the most common neoplasmic disease in Korea. It is related to the Korean lifestyle and there is possibility of its recurrence, people with cervical cancer need to lead a healthy lifestyle $[1,2]$. As the number of colon cancer patients and their death rate increases, this study is to understand the occurrences in patients with hysterectomy in Korea and to provide active help for treatment, prevention and management of hysterectomy patients The most common treatment of cervical cancer was hysterectomy which takes up $38.1 \%$ of cervical cancer patients stood out from other cancer treatment $[1,3,4]$.

The incidence of cervical cancer has been reported in Eastern and Western countries. Cervical cancer is reported to be the most common malignant neoplasm in Asia, specifically, China, Japan, and Korea. In contrast, cervical cancer incidence in the United States among Caucasians is low and among blacks, moderate to low. Only one other study has directly compared the rates of cervical cancer in the three ethnic groups (i.e., white, African American, and immigrant Korean) living in the same region. Cervical cancer is that shows increasing trend and in need of appropriate countermeasures and managements for decreasing cancer risk. The authors extend their investigation by comparing the incidence rate of cervical cancer among the same three ethnic groups in the state of Illinois from 2006 to 2008. In this study, the incidence of cervical cancer was observed to be lowest in whites, intermediate in African Americans, and highest in immigrant Koreans. The overall 3year cumulative incidence rate from 2006 to 2008 was $63.2 / 100,000(95 \%$ confidence interval (Cl 41.9-85.3), 39.5/100,000(95\% Cl 36.8-42), and 47.6/100,000 (95\% Cl 31.858.5) for immigrant Koreans, African Americans, and Whites, respectively. The 3 -year 
age-adjusted cumulative incidence rate for immigrant Koreans(174/100,000) was approximately four-and eightfold higher than for African Americans $(53 / 100,000)$ and whites $(41 / 100,000)[4,5,6]$.

The incidence of, and indications for cervical cancer in Finland are approximately the same as in other European countries, but hysterectomy patients is more common in Finland. Because the surgery for the 'uteri or cervix at risk' population produced charges in the rates of hysterectomy, the hysterectomy should be considered when calculating cervical cancer rates or incidences of cervical or endometrial cancer. Postoperative nausea, vomiting and depression are a 'Big Little Problem' during the period of recovery after hysterectomy [7, 8].

Therefore the causes and prevention of cervical cancer analysis, an appropriate management program is required. Screening examinations for early cervical cancer detection include a cervical examination, myoma examination by specialist and Pap smear $[9,10]$. According to the previous research related to prevent cervical cancer, optimal methods such as education-based method, cancer screening and enumerative schemes have been typically used to prevent cervical cancer. However, these methods have problem taking a lot of time to search for optimal cervical cancer prevention. Also, since the previous research did not consider the initial cost as a criterion along with the stage of cervical cancer, it is difficult to draw cost-effective method. Accordingly, it is necessary to select an optimal combination which derives maximum effect within allowable cost when making a cervical patient plan during hysterectomy of cancer patients. When these methods are combined complementarily, the effectiveness could be more enhanced.

In this regard, this study was attempted to develop model program regarding information system and its effectiveness. The model can be utilized variously in health management in the respect that it helps to manage comprehensively the construction information and make reliable decision. In addition, the adoption of model is sufficient in the health area. The purpose of this study is to develop work process models and their guides in order to utilize model effectively in health work at construction stage. The model can apply to hysterectomy patients by analyzing the system and process. This study developed an effective information model by constructing the existing health status in connection with health function through an analysis on the relationship between health function and physical work, and constructing the role of each project participants. In order to improve the usefulness of the developed models, guides that described the health works of project participants were prepared through interviews and case studies. To validate the utilization of the models, a comparative analysis on the information process of precedent studies was also made and a survey was conducted on experts. This study can contribute to increasing the utilization of cervical cancer patients in the health area and can be helpful to develop the model for hysterectomy patients. In the future, it will be necessary to make an assessment on the model from the results through case applications and constantly update information-based model in consideration of the improvement of health behaviors due to the application of information.

There has been an increasing trend in hysterectomy every year. They receive one or more treatments such as chemotherapy, radiation treatment and hormone therapy after hysterectomy $[10,11]$. However, most patients suffer from depression after hysterectomy. In order to help the health improvement of hysterectomy patients, there is the need to develop the empirical model for the prevention of depression after hysterectomy. In order to solve the problem, we should look for the practical plans. There were few studies to deal with information application of predictive model for health promotion in patients with hysterectomy until present in Korea. Thus, we need to develop depressive program for hysterectomy patients. 
Therefore, this study was attempted new intervention strategies for depression prevention after hysterectomy to promote health behaviors that are constructive to a healthy lifestyle. This study developed the role of empirical model in comprehensive and systematical prevention and treatment of depression with hysterectomy patients. Statistical significance for the depression prevention after hysterectomy will be a help to prevention of cervical cancer. This study designed to develop the long-term health program and ultimately to analyze the intervention effect through its application. This is to identify the effective development of empirical model by analyzing the change in health behaviors by intervention for the purpose of preventing their depression.

The purpose of this study is to conduct a comparative verification on the application of empirical program suitable for hysterectomy patients to prevent their depression. Because the study has verified the effect of an information program to boost hysterectomy patients through a scientific measurement method, it is expected that the study will help with an improvement in the quality of life through application of the empirical model to solve problems from increasing hysterectomy patients and related depression.

\section{Materials and Methods}

\subsection{Construction of Empirical Model}

This research is composed of three-step basic strategies [Figure 1]. The first success factor of the system is subjects' lack recognition for cervical cancer prevention which is the biggest problem of the hysterectomy patients. The strategies of the second-stage are program which selected the order of priority of the time and the importance in the limitation of the budget and the practical implementation. The strategies of third-stage are the effect evaluation on the empirical performance for the depression prevention of patients with hysterectomy [Figure 2].

\subsection{Materials}

The materials performed to investigate the effect of empirical model, the data were collected from patients undergone a hysterectomy in a university located in D city from July 1 , 2013 to October 10, 2013. 134 patients undergoing a hysterectomy were randomly allocated to two groups. The experimental group (67 patients) who did not have depression and control group(67 patients) who have depression. The program was held 4 times per week for 100 days [Table 1].

The instrument for testing patients undergoing depression with hysterectomy consists of a total of 22 items. The first items for general characteristics are consisted 7 items of age, marital status, economic status, educational level, other disease, occupation, family cancer history. The second items for health practice rate for a depression prevention are consisted 15 items of perceived stress, exercise, body weight control, meat intake, cholesterol control, depression status, hypertension, blood sugar control, music listening, vegetable intake, passive smoking, drinking alcohol, intellectual activity, sexual intercourse, sexual satisfaction.

\subsection{Study Methods}

All statistical analyses were carried out using SAS package program (version 10.0 for Windows) for descriptive analysis, $\mathrm{X}^{2}$-test, t-test, and importance-performance analysis.

General characteristics of study subjects were measured by percentage and number. $\chi^{2}$-test was used to determine the general characteristics of the subjects including age. In addition, general characteristics of study subjects were measured by percentage and number. The 
pairwise t-test was done to the before and after application for empirical performance rate of a depression prevention program. This was conducted to observe some significant differences between the two groups before and after the application of the empirical model. The statistical significant level was set was at 0.05 .
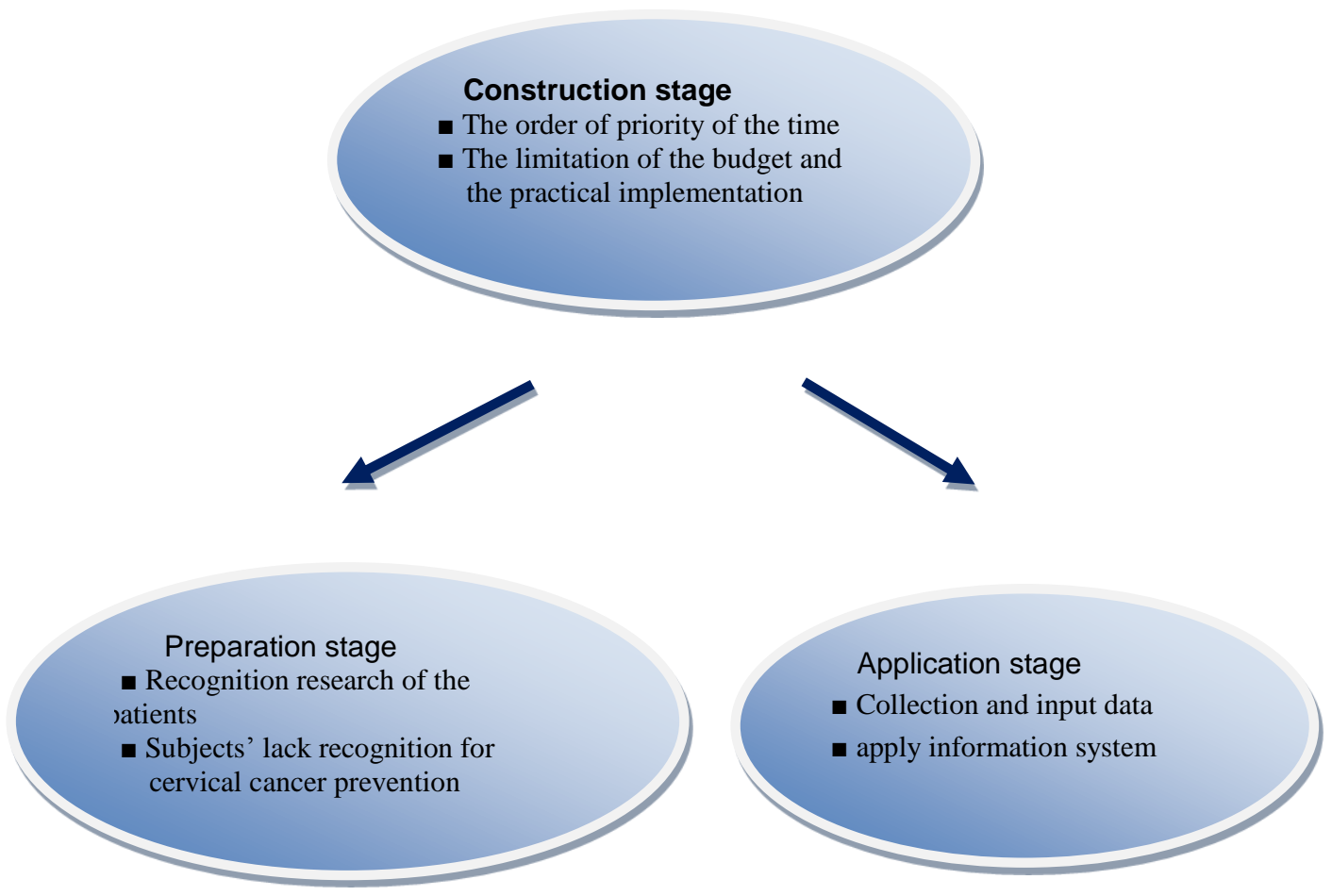

Evaluation stage

- Effect evaluation on the empirical

performance for depression atients

information system

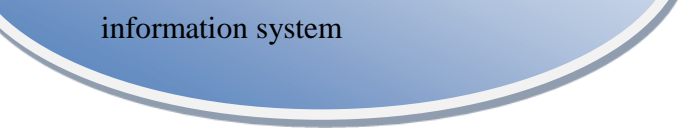

Figure 1. Construction of Empirical Model for the Depression with Hysterectomy 


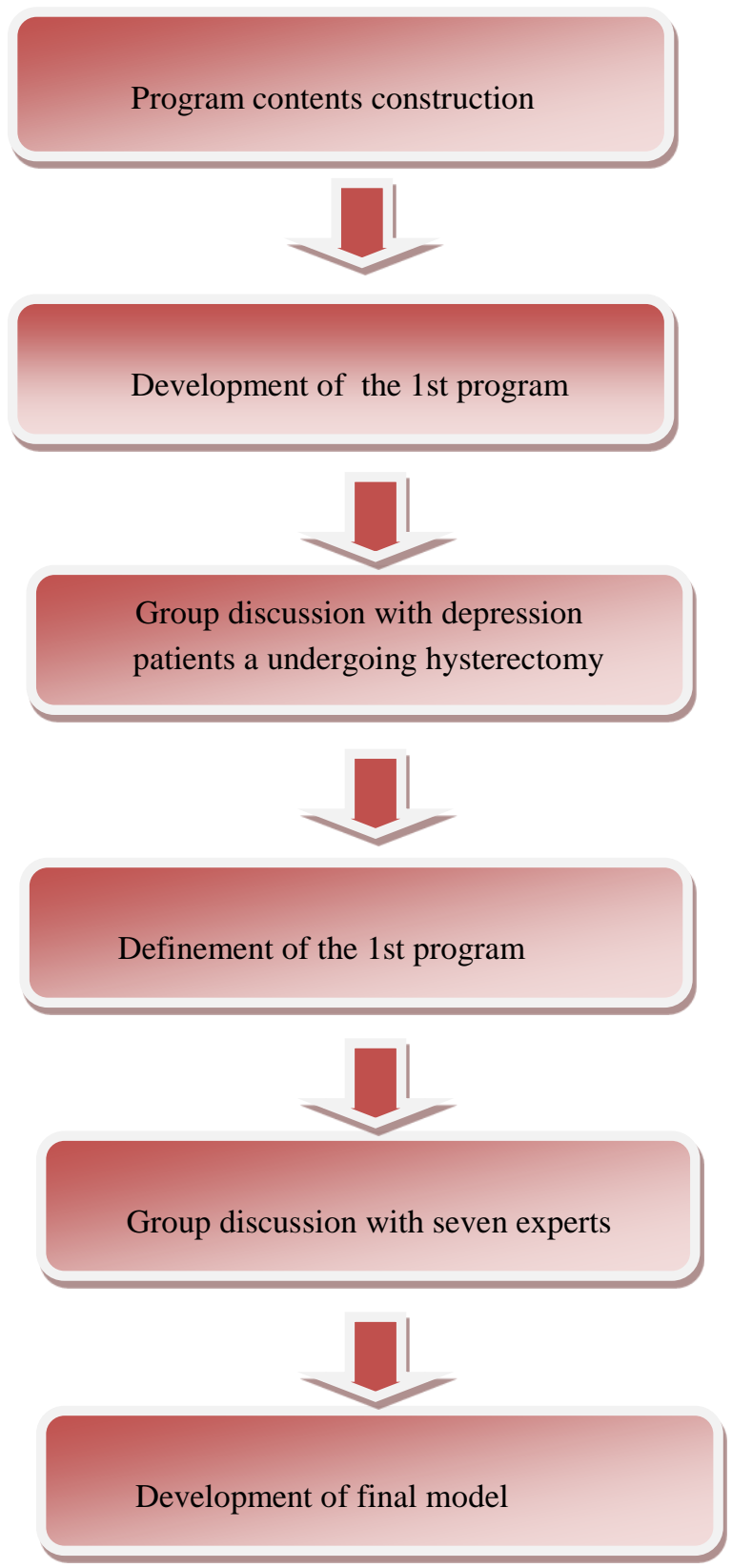

Figure 2. Process of Development of Efficient Information Model

Table 1. Comprehensive Information Program

\begin{tabular}{lc}
\hline Session & Duration \\
\hline Causes, treatments and prognosis & 1 hour \\
Diet of patients with hysterectomy & $30 \mathrm{~min}$ \\
Purpose, self-care and cervical self-examination & $30 \mathrm{~min}$ \\
Exercise after operation & 1 hour \\
Side-effects, stress management & $30 \mathrm{~min}$ \\
Health practice & 1 hour \\
\hline
\end{tabular}




\section{Result}

\subsection{General Characteristics of Study Subjects}

Table 2 presents general characteristics of study subjects. Comparing age of the both group, patients aged from 40-49's were higher in the experimental group (35.8\%) than the control group $(28.4 \%)$. In terms of marital status, the response rate $(73.1 \%)$ of married women was lower in the experimental group than the response rate $(79.1 \%)$ of the control group. The response rate $(41.8 \%)$ of experimental group with other disease was a statistically significantly higher than the response rate $(23.9 \%)$ of the control $\operatorname{group}\left(\mathrm{X}^{2}=5.39, \mathrm{p}=.027\right)$ according to other diseases of subjects. On the other hand, for subjects with a family history, the response rate $(26.9 \%)$ of experimental group was a statistically significantly higher than the response rate $(13.4 \%)$ of the control group $\left(\mathrm{X}^{2}=3.58, \mathrm{p}=.041\right)$.

Table 2. General Characteristics of Study Subjects

\begin{tabular}{|c|c|c|c|c|}
\hline Variables & $\begin{array}{c}\text { Experimental } \\
\text { Group }\end{array}$ & $\begin{array}{c}\text { Control } \\
\text { Group }\end{array}$ & $\mathrm{X}^{2}$ & $\mathrm{P}$ \\
\hline \multicolumn{5}{|l|}{ Age } \\
\hline$\leq 39$ & 11(16.4) & $15(22.4)$ & 10.82 & .075 \\
\hline $40-49$ & $24(35.8)$ & $19(28.4)$ & & \\
\hline $50-59$ & $19(28.4)$ & $23(34.3)$ & & \\
\hline$\geq 60$ & $13(19.4)$ & $10(14.9)$ & & \\
\hline \multicolumn{5}{|l|}{ Marital status } \\
\hline Single & $18(26.9)$ & $14(20.9)$ & 6.15 & .692 \\
\hline Married & $49(73.1)$ & $53(79.1)$ & & \\
\hline \multicolumn{5}{|l|}{ Economic status } \\
\hline Less than 2 millions & $23(34.3)$ & 21(31.3) & 13.94 & .805 \\
\hline 2-2.99 millions & $19(28.4)$ & $14(20.9)$ & & \\
\hline 3-4.99 millions & $14(20.9)$ & $19(28.4)$ & & \\
\hline Above 5 millions & $11(16.4)$ & $13(19.4)$ & & \\
\hline \multicolumn{5}{|l|}{ Educational } \\
\hline Less than middle s. & $10(14.9)$ & $14(20.9)$ & 11.82 & .174 \\
\hline High school & $25(37.3)$ & $28(41.8)$ & & \\
\hline Above college & $32(47.8)$ & $25(37.3)$ & & \\
\hline \multicolumn{5}{|l|}{ Other disease } \\
\hline Yes & $28(41.8)$ & $16(23.9)$ & 5.39 & .027 \\
\hline No & $39(58.2)$ & $51(76.1)$ & & \\
\hline \multicolumn{5}{|l|}{ Occupation } \\
\hline Yes & $38(56.7)$ & $30(44.8)$ & 9.26 & .353 \\
\hline No & $29(43.3)$ & $37(55.2)$ & & \\
\hline \multicolumn{5}{|l|}{ Family history } \\
\hline Yes & $18(26.9)$ & $9(13.4)$ & 3.58 & .041 \\
\hline No & $49(73.1)$ & $58(86.6)$ & & \\
\hline Total & $67(100.0)$ & $67(100.0)$ & & \\
\hline
\end{tabular}




\subsection{Comparison of Health Practice Rate for a Depression in Hysterectomy Patients}

Table 3 presents the comparison of health practice rate for a depression prevention in hysterectomy patients before and after intervention. Below table notes, the mean score $(59.47 \pm 1.65)$ of the degree of perceived stress after intervention was a statistically significantly lower score than the mean score $(72.51 \pm 0.83)$ of respondents before the intervention( $\mathrm{t}=.017, \mathrm{t}=2.81)$. The mean $\operatorname{score}(47.25 \pm 0.39)$ of the respondents who do regularly exercise after intervention was a significantly higher score than the mean $\operatorname{score}(35.19 \pm 1.45)$ of respondents before intervention $(\mathrm{t}=-5.27, \mathrm{p}=.042)$. The mean score $(56.89 \pm 0.47)$ of the degree of depression status after intervention was a statistically significantly decreased score than the mean score $(74.29 \pm 0.42)$ of $\operatorname{subjects}(\mathrm{t}=2.08, \mathrm{p}=.015)$ before the intervention. And then behavioral change(62.74 \pm 0.26$)$ of sexual intercourse after intervention was a significantly higher score than behavior change $(41.56 \pm 0.84)$ before the intervention $(\mathrm{t}=-4.91, \mathrm{p}=.036)$.

Table 3. Comparison of Health Practice Rate for a Depression Prevention

\begin{tabular}{|c|c|c|c|c|}
\hline \multirow[b]{2}{*}{ Variables } & Before & After & & \\
\hline & Mean \pm S.D. & Mean \pm S.D. & $\mathrm{t}$ & $\mathrm{P}$ \\
\hline Perceived stress & $72.51 \pm 0.83$ & $59.47 \pm 1.65$ & 2.81 & .017 \\
\hline Exercise & $35.19 \pm 1.45$ & $47.25 \pm 0.39$ & -5.27 & .042 \\
\hline Body weight control & $41.65 \pm 1.72$ & $43.18 \pm 1.64$ & -6.92 & .798 \\
\hline Meat intake & $47.14 \pm 0.54$ & $39.76 \pm 0.82$ & 8.14 & .136 \\
\hline Cholesterol control & $62.37 \pm 1.56$ & $42.13 \pm 2.61$ & 1.35 & .042 \\
\hline Depression status & $74.29 \pm 0.42$ & $56.89 \pm 0.47$ & 2.08 & .015 \\
\hline Hypertension & $69.17 \pm 1.85$ & $61.36 \pm 1.62$ & 6.24 & .579 \\
\hline Blood sugar control & $71.06 \pm 1.74$ & $57.29 \pm 0.45$ & 2.67 & .034 \\
\hline Music listening & $39.58 \pm 0.26$ & $45.17 \pm 0.54$ & 1.56 & .045 \\
\hline Vegetable intake & $51.46 \pm 0.91$ & $69.32 \pm 0.08$ & -4.42 & .173 \\
\hline Passive smoking & $38.72 \pm 0.60$ & $27.54 \pm 0.62$ & 0.36 & .092 \\
\hline Drinking alcohol & $63.49 \pm 0.28$ & $47.22 \pm 0.57$ & 2.81 & .046 \\
\hline Intellectual activity & $62.15 \pm 0.67$ & $74.16 \pm 1.52$ & -1.69 & .015 \\
\hline Sexual intercourse & $41.56 \pm 0.84$ & $62.74 \pm 0.26$ & -4.91 & .036 \\
\hline Sexual satisfaction & $52.18 \pm 0.52$ & $68.52 \pm 0.82$ & -2.36 & .041 \\
\hline
\end{tabular}

\subsection{The Change of Health Practice Performance between Two Groups}

Figure 3 presents the change of health practice performance before and after information application of a model between two groups. The information application in both experimental and control groups was improved in hypertensive control. On the other hand, the experimental group had more significant improvement in blood surgar control but no change in body weight control detected in both groups. 


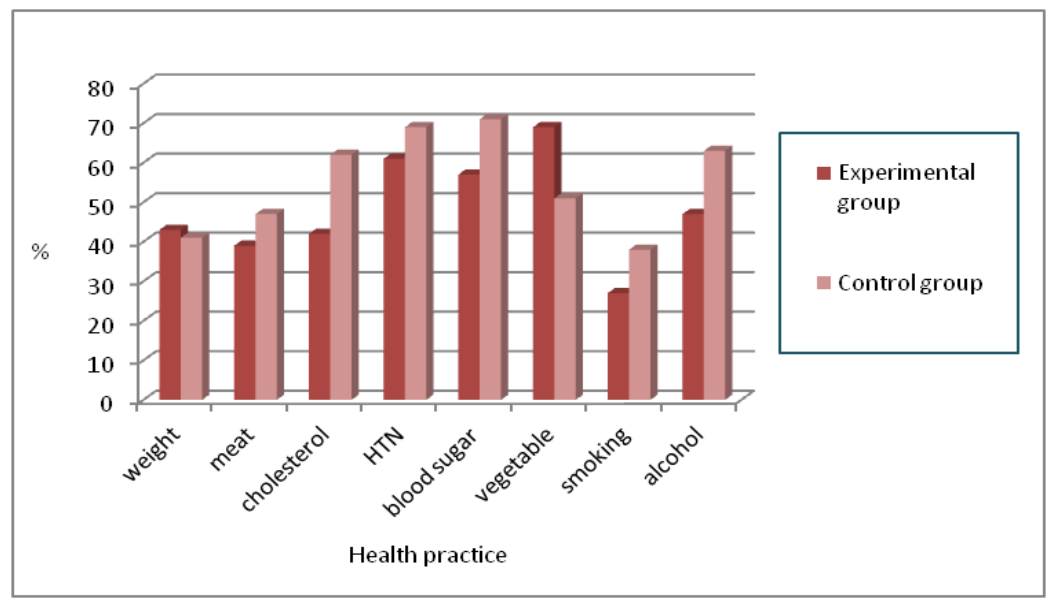

Figure 3. The Change of Health Practice in Two Groups

\subsection{The Change of Life Habit Before and After Information Application}

Figure 4 presents the change of life habit before and after information application in two groups. The information application in both experimental and control groups in the life habit was improved. The sexual satisfactions in hysterectomy patients by intervention were a significantly higher rate in the experimental group compared to the control group ( $\mathrm{p}<.05)$. Individual perceived stress was significantly decreased compared with the control group $(\mathrm{p}<.05)$. Additionally, activities with music listening in patients undergoing hysterectomy were higher in the experimental group than the control group.

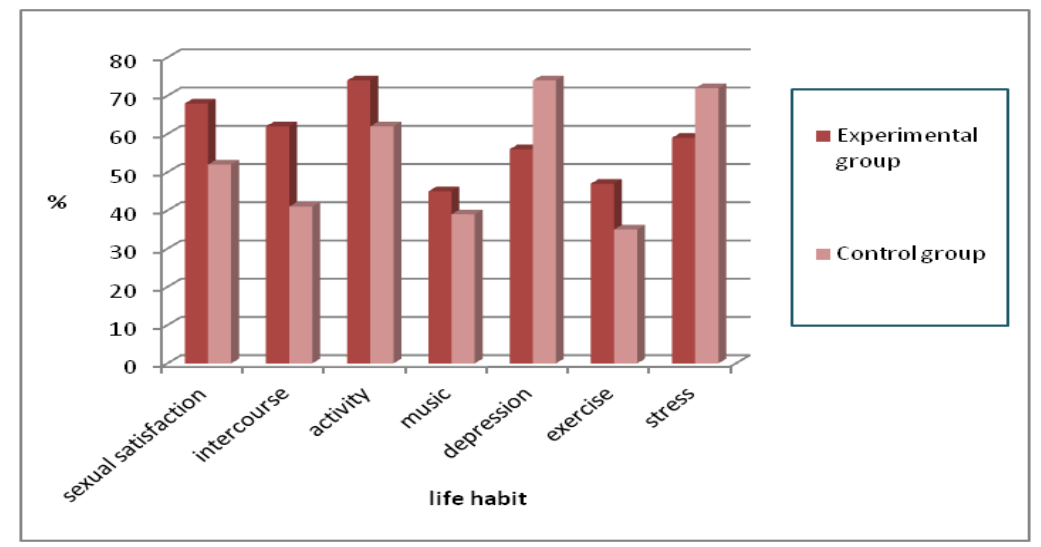

Figure 4. The Change of Life Habit in Two Groups

\subsection{Durability of the Clinical Performance Rate by Application of a Model}

Figure 5 presents the effect of durability of clinical performance rate by application of a model between the two groups. Application effect of the feedback system was higher in the experimental group than the control group, regardless of the time elapsed of 60 days after application of feedback system. However, the application effect decreased rapidly with time elapsed of 80 days after application in the experimental group as compared to the control group. 


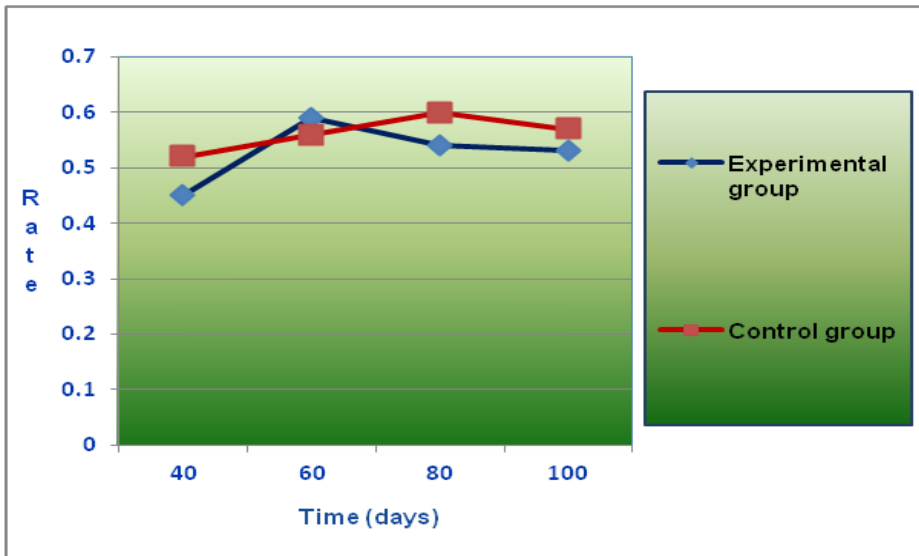

Figure 5. Durability of the Clinical Performance Rate by Application of a Model

The slope is often called delta $\mathrm{x} /$ delta $\mathrm{y}$. This change in $\mathrm{x} /$ change in $\mathrm{y}$. To actually calculate the $\mathrm{y}$ value of the triangle edge for a given integer value of $\mathrm{x}$, as we move incrementally along the $\mathrm{x}$ axis one pixel at a time, we use the slope value [1].

$*$ Slope $=\frac{\triangle Y}{\triangle X}$ Where $\triangle \mathrm{X}:$ time interval
$\triangle \mathrm{Y}:$ variation of intervention effect

$*$ Ratio $=\triangle \mathrm{Ya}$ Where $\triangle \mathrm{Yb}$ : clincial performance rate before $\triangle \mathrm{Yb} \quad$ application

$\triangle$ Ya : clinical performance rate after application

\section{Discussion}

This study was attempted to find out the effect of the empirical model for the prevention of depression in patients undergoing a hysterectomy.

As a result, stress control was significantly decreased after intervention in subjects compared with before of the intervention. The results showed that clinical information significantly effect in reducing depression and in increasing sexual satisfaction of the experimental group. The significance of health practice performance on the activities of subjects' music listening showed after intervention as compared before intervention. The finding was consistent with the result of earlier researches $[12,13]$. These findings suggest that the application of clinical information system may be effective to reduce the stress and increase the intellectual and emotional activity. This paper also shows the feasibility of the system through some tests. This study will provide basic materials for health mediation program to enhance the quality of life of women after hysterectomy by identifying the change of psychiatric status, the sexual life and sexual satisfaction of depressive patients after hysterectomy. Based on the results, the empirical model may be recommended as a useful intervention to effectively reduce the levels of depression, anxiety and pain among patients with hysterectomy. In the future, it is proposed to perform diversified studies to enhance the psychological status, healthy life and improvement of sexual satisfaction of the women after hysterectomy and to develop education and counseling programs. The present results suggest that the development of empirical model would play the health promotion role against depression. 
The health habit is the most important factor for the impact of psychological status on the physical stress and depression in patients with hysterectomy [15]. We suggest this study provides helpful reference to implement effective health intervention to keep psychological status within normal limits after short time for hysterectomy patients. Therefore surgeons should concern about the postoperative psychological supports for the patients with hysterectomy. These results suggest that the knowledge and faith could reduce postoperative stress and depression of the patients with hysterectomy and significantly affected the improvement of their depression coping strategies. Concern could be a psychological program for management of postoperative stress and depression in patients with hysterectomy.

Therefore, on an implementation of health practice performance in hysterectomy patients, the results of proposed methods are expected to contribute to the efficient model development and information system.

\section{Conclusion}

The purpose of this study is to conduct a comparative verification on the application of empirical program suitable for hysterectomy patients to prevent their depression.

The data were collected from patients undergone a hysterectomy in a university located in D city from July 1, 2013 to October 10, 2013. 134 patients undergoing a hysterectomy were randomly allocated to two groups. The experimental group(67 patients) who did not have depression and control group(67 patients) who have depression. The program was held 4 times per week for 4 weeks. All statistical analyses were carried out using SAS package program for descriptive analysis, $\mathrm{X}^{2}$-test, $\mathrm{t}$-test, and importance-performance analysis. The results of this paper are follows.

Firstly, the response rate $(41.8 \%)$ of experimental group with other disease was a statistically significantly higher rate than the response rate(23.9\%) of the control group $\left(\mathrm{X}^{2}=5.39, \mathrm{p}=.027\right)$ according to other diseases of subjects. On the other hand, for subjects with a family history, the response rate(26.9\%) of experimental group was a statistically significantly higher rate than the response rate(13.4\%) of the control $\operatorname{group}\left(\mathrm{X}^{2}=3.58, \mathrm{p}=.041\right)$.

Secondly, the mean score $(56.89 \pm 0.47)$ of the degree of depression status after intervention was significantly decreased than the mean $\operatorname{score}(74.29 \pm 0.42)$ of $\operatorname{subjects}(t=2.08, p=.015)$ before the intervention. And then behavioral change $(62.74 \pm 0.26)$ of sexual intercourse after intervention was significantly higher than behavior change(41.56 \pm 0.84$)$ before the intervention $(\mathrm{t}=-4.91, \mathrm{p}=.036)$.

Thirdly, the sexual satisfaction in hysterectomy patients by intervention was significantly higher in the experimental group compared to the control group $(\mathrm{p}<.05)$. Individual perceived stress was decreased significantly compared with the control group $(\mathrm{p}<.05)$. Activities with music listening in patients undergoing hysterectomy were higher in the experimental group than the control group.

Fourthly, application effect of the feedback system was higher in the experimental group than the control group, regardless of the time elapsed of 60 days after application of feedback system. However, the application effect decreased rapidly with time elapsed of 80 days after application in the experimental group as compared to the control group.

Fifthly, the present research showed that the empirical performance rate of patients with hysterectomy can be increased to $68.5-74.2 \%$ by the model application.

In conclusion, the level of depression in female patients undergoing a hysterectomy has clinical relevance to knowledge and faith about hysterectomy. Thus, faith and health practice should be applied to the development of an effective information intervention program. It is 
necessary to make them check regularly by building the practice management system of the cervical prevention, making them recognize the existence, making them participate in the practice program of rules for the cancer prevention, and making them apply also the interactive communication frame. The results of application of this information system showed as an appropriate model. The model can be used as an effective method to reduce of depression for patients with hysterectomy.

\section{References}

[1] Statistics Korea, Annual Report on the Cause of Death Statistics, (2013).

[2] A. M. Fitzgerald-Smith, P. Shrivastava and J. Hershman, "The Role of the Nurse in Colorectal Cancer Follow-up", Hospital Medicine, vol. 64, no. 6, (2003), pp. 344-347.

[3] H. Wu, Q. Lin, B. Jin and Q. Liu, "Application of Improved DBSCAN Algorithm in the Plan Compilation Management”, IJDTA, vol. 5, n o.23, (2012), pp. 32.

[4] S. C. Galloway and J. E. Graydon, "Uncertainty Symptom Distress and Information Needs after Surgery for Cancer of the Colon", Cancer Nursing, vol. 19, no. 2, (2006), pp. 112-117.

[5] W. G. Ouchi, B. Markets and W. Royce, "Software Project Management, A Unified Framework", AddisonWesley, (2001).

[6] A. Kazi, D. M. Smith, K. Daniel, S. Zhong, P. Gupta and M. E. Bosley, "Potential Molecular Targets of Tea Polyphenols in Human Tumor Cells", Significance in Cancer Prevention, in Vivo, (2002), pp. 397-403.

[7] S. C. Galloway and J. E. Graydon, "Uncertainty Symptom Distress and Information Needs after Surgery for Cancer of the Colon", Cancer Nursing, vol. 19, no. 2, (2006), p. 112-117.

[8] C. S. Yang and X. Wang, “Green Tea and Cancer Prevention”, Nutr Cancer, vol. 62, (2010), pp. 931-937.

[9] A. M. Davis, “A Strategy for Comparing Alternative Software Development Life Cycle Model”, IEEE Trans, Software Eng, (2008), pp. 1453-1461.

[10] M. Rad, A. Attar and R. Ebrahimi Atani, “A Comprehensive Layer Based Encryption Method for Visual Data”, IJSIP, vol. 6, no. 1, (2013), pp. 13-48.

[11] W. G. Ouchi, "A Conceptual Framework for the Design of Organizational Control Mechanisms", Management Science, vol. 25, no. 9, (2009), pp. 833-848.

[12] K. J. Mayer and R. M. Salomon, "Capabilities, Contractual Hazards, and Governance, Integrating, Resourcebased and Transaction Cost Perspectives”, Academy of Management Journals, vol. 2, no. 6, (2006), pp. 942959.

[13] B. Tuttle, A. Harrell and P. Harrison, "Moral Hazard Ethical Considerations and the Decision to Implement an Information System", Journal of Management Information Systems, vol. 13, no. 4, 7, (2007), pp. 25.

[14] W. XiaoPeng, W. Bailing, L. Yang, H. Hui and S. Yunxiao, "A New Ecology Model for Internet Worm Security Threat Evaluation”, IJFGCN, vol. 6, no. 2, (2013), pp. 107-116.

[15] S. R. Lee, "Workshop on Bioscience and Medical Research 2014", Third, Jeju Island, Korea, (2014) April $15-18$.

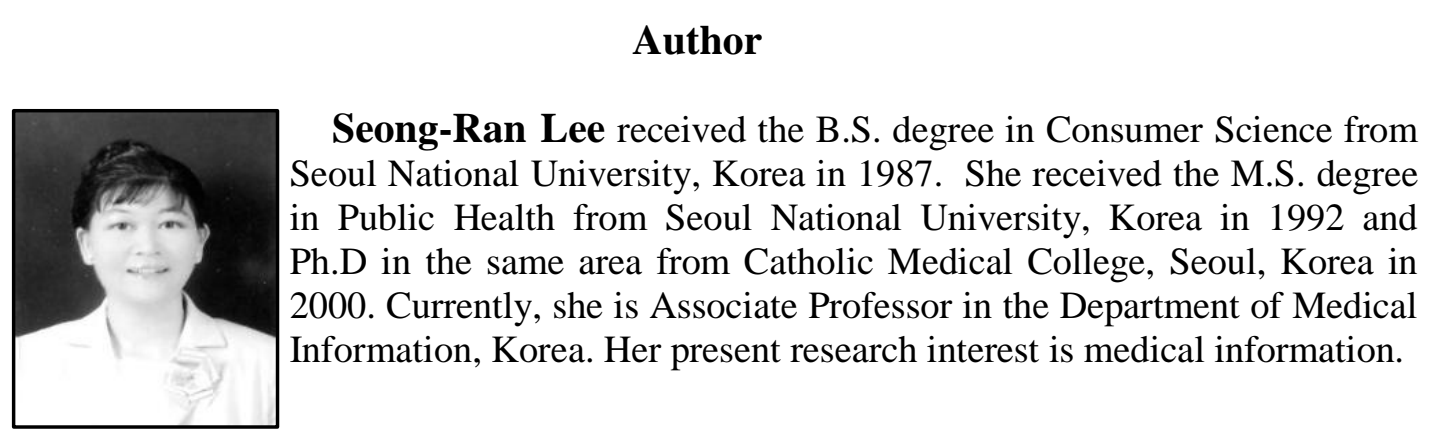


International Journal of Bio-Science and Bio-Technology Vol.6, No.4 (2014) 